\title{
Identification of the Parathyroid Gland with Vasculature by Intraoperative Carbon Nanoparticles
}

\author{
Chuanchang Yin ${ }^{*}$, Bin Song1, Xiaoyan Wang ${ }^{2}$ \\ ${ }^{1}$ Department of Thyroid Surgery, Jingzhou No. 1 People's Hospital and First Affiliated Hospital of Yangtze University, Jingzhou, \\ China \\ ${ }^{2}$ Department of Pathology, Jingzhou No. 1 People's Hospital and First Affiliated Hospital of Yangtze University, Jingzhou, \\ China \\ Email: *yinchuanchang@yahoo.com
}

How to cite this paper: Yin, C.C., Song, B. and Wang, X.Y. (2021) Identification of the Parathyroid Gland with Vasculature by Intraoperative Carbon Nanoparticles. Yangtze Medicine, 5, 79-89. https://doi.org/10.4236/ym.2021.52009

Received: March 30, 2021

Accepted: April 12, 2021

Published: April 15, 2021

Copyright $\odot 2021$ by author(s) and Scientific Research Publishing Inc. This work is licensed under the Creative Commons Attribution International License (CC BY 4.0).

http://creativecommons.org/licenses/by/4.0/ (c) (i) Open Access

\begin{abstract}
Background: We aimed to investigate the ability of carbon nanoparticles to identify parathyroid glands with vasculature during thyroid surgery. Material and methods: Totally 42 patients with various thyroid diseases were selected for the prospective research of carbon nanoparticle injection used in thyroidectomy. Another 42 patients without receiving carbon nanoparticle injection were selected as the control group. All cases underwent total or subtotal bilateral thyroidectomy. Before the ligation of the superior and inferior poles of the thyriod lobes, $0.1 \mathrm{~mL}$ of a carbon nanoparticle suspension was injected into the two poles. Important tissues such as the recurrent laryngeal nerve, parathyroid gland with blood supply and the inferior thyroid artery were identified and protected. Results: The parathyroid glands with vasculature were not stained and thus remained the primary color in all cases, while abnormal thyroid tissues were stained black. After 5 minutes, thyroid lobes were injected with the carbon nanoparticle suspension and the original color of parathyroid glands was unchanged. Even the parathyroids with blood supply can be identified and protected. The number of parathyroid glands for autotransplantation was one in the test group and six in the control group. At one day after the thyroid surgery, hypocalcemia became detectable in four patients of the test group and in five of the control group. Twenty-four instances of hypoparathyroidism occurred at 1 day after surgery in the control group, while ten instances in the test group. Conclusion: Intraoperatvie carbon nanoparticle suspension injection is an effective and safe technique for guiding thyroid surgery. The carbon nanoparticle suspension plays an important role in identifying the vasculatural parathyroid glands, while protecting the physiologic function of the parathyroid glands during surgery.
\end{abstract}




\section{Keywords}

Carbon Nanoparticle Suspension (CNPs), Parathyroid Gland (PTG), Thyroidectomy, Hypoparathyroidism

\section{Introduction}

Identification of the parathyroid gland (PTG) is one of the most important parts during the parathyroid and thyroid surgery. The main cause for postoperative hypocalcemia complication is the iatrogenic injury and inadvertant excision of PTG or vasculature. About $9.9 \%$ of thyroid patients undergoing surgical treatment suffer from postoperative hypocalcemia for parathyroid dysfunction according to a multicenter survey of Europe [1]. Until now, identification and preservation of PTGs have mostly relied on the experience of surgeons [2]. The effective methods for identification of PTGs are parathyroid hormone (PTH) assay and pathyologicalexamnation [3]. However, accurate and quick intraoperative localization of PTGs is the key point to protect vasculatural PTG for minimizing postoperative complications and improve the quality of life of patients. Recently, Jindong Li et al. found a negative staining method of PTGs through carbon nanoparticle (CNPs) diffusion in the lymphatic vessels [4].

In our study, we aimed to safely identify and protect PTGs and blood supply by using CNPs suspension in thyroid surgery. Herein, we demonstrate an easy and rapid negative staining approach for intraoperative identification of vasculatural PTGs using a ready-made CNPs suspension (0.5 $\mathrm{ml}$ ampol, Lai Mei Pharmaceutical Co., Chongqing, China).

\section{Materials and Methods}

\subsection{Patients}

This clinical study was approved by the local ethic committee. All informed consents were signed by the patients before surgery. Between January 2017 and January 2020, 84 thyroid tumor patients underwent thyroid surgery at the First Affiliated Hospital of Yangtze University (Table 1). Surgical indications for treatment were as follows: thyroid carcinoma $(n=47)$ and benign thyroid tumor $(n=37)$. Exclusion criteria were as follows: preoperative neck surgery, preoperative hypoparathyroidism or hypocalcemia, and neck radiotherapy. An experienced operation team conducted all the surgeries with the general anesthesia. Serum calcium and PTH levels were checked before surgery to assess hypoparathyroidism or hypocalcemia. Hypocalcemia was defined as serum calcium level $<2 \mathrm{mmol} / \mathrm{l}$ (normal range $2-2.8 \mathrm{mmol} / \mathrm{l}$ ) with oral paresthesia or laryngospasm [5]. The serum PTH level $<15 \mathrm{pg} / \mathrm{ml}$ (normal range $15-68.3 \mathrm{pg} / \mathrm{ml}$ ) means hypoparathyroidism [6] [7]. The clinical manifestations of hypocalcemia were recorded, such as laryngospasm, seizure and oral paresthesia. Pathological findings 
Table 1. All patients' demographic features of patients in two groups.

\begin{tabular}{cccc}
\hline & Nanocarbon particles group & Control group & P value \\
\hline Age (y) & $51.57 \pm 1.79$ & $52.88 \pm 1.77$ & 0.603 \\
Gender & 32 & 33 & 0.794 \\
female & 10 & 9 & \\
male & & & 0.272 \\
Type of surgery & 26 & 21 & \\
Total thyroidectomy & 16 & 21 & \\
Subtotal thyroidectomy & & & \\
Surgical cause & 26 & 21 & \\
Cancer & 16 & 21 & \\
Non-cancer & &
\end{tabular}

of PTG, and serum PTH and calcium levels were recorded independently both before and after surgey.

\subsection{Intraoperative Identification of PTG}

All patients underwent total thyroidectomy with a trandionally low-collar incision. For each patient, the anterior muscles of the neck were retracted to expose the surgical thyroid lobe (Figure 1). Without excessive dissection, a nanocarbon suspension $(0.5 \mathrm{ml}$ ampol) was slowly injected into the abnormal lobe using a $1-\mathrm{mL}$ syringe with a skin test needle in the upper and lower poles of the gland ( $0.1 \mathrm{ml}$ each pole, if the abnormal thyroid was small, the injection point was only one). Before injecting the suspension, we pulled back on the syringe to avoid the injection of CNPs into the blood vessels (Figure 1). Then the puncture point was gently pressed for 1 minute with gauze. We can observe and localize the PTG after the nanocarbon injection, despite the hardness to find and the tiny size. Tissues, such as the vascular PTG, were identified and dissected (Figures 2-4), while the thyroid gland was stained black. The superior pole was ligated and cut close to the thyroid capsule by preserving the posterior branches of superior thyroid artery (STA) for superior PTG after the gland developed completely within 3 - 5 minutes. The terminal branches of the inferior thyroid artery (ITA) were preserved for the inferior parathyroid after cutting of ITA (Figure 2). If the frozen-section diagnosis was confirmed as thyroid cancer, total thyroidectomy with central lymphy node dissection was performed.

\section{Statistical Analysis}

Data were analyzed by SPSS 23.0. The preoperative and postoperative data were expressed as mean \pm standard deviation $(\mathrm{X} \pm \mathrm{s}$ ) for analysis, and compared between groups using Student's $t$ test and the $\chi^{2}$ test. When $\mathrm{P}$ was $<0.05$, the variance between preoperative and postoperative data of the two groups was significant. 


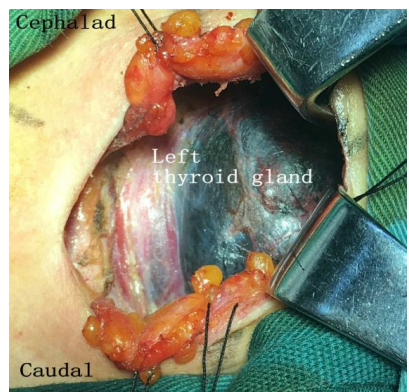

Figure 1. After carbon nanoparticles suspension injected. We retracted the anterior muscles of neck to expose the surgical thyroid lobe. Without excessive dissection, the nano-carbon suspension ( $0.5 \mathrm{ml}$ ampol, Lai Mei Pharmaceutical Co, Chongqing, China) was slowly injected into the abnormal lobe using 1-mL syringe with a skin test needle in the upper and lower pole of gland $(0.1 \mathrm{ml}$ each pole, if the abnormal thyroid is small, the injection point only one). Before injecting the suspension, we pull back on the syringe to avoid the carbon nanoparticles injected into the blood vessels. Then, the puncture point should be gently pressed for 1 minutes with gauze. For unexperienced surgeons, these procedures are easily done.

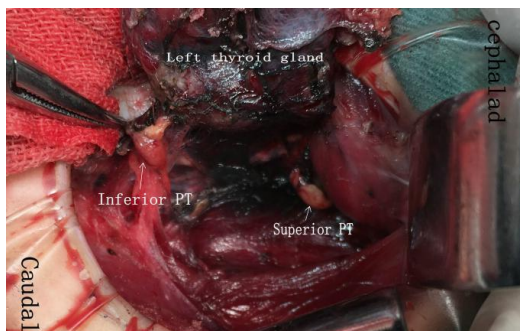

Figure 2. Five minutes after carbon nanoparticles suspension injected ( $\downarrow$ un-developed superior and inferior parathyroid gland). PT: parathyroid gland. We could observe the parathyroid gland and identify its location after the nano-carbons injection, despite its being hard to find and tiny. Tissues, such as the parathyroid gland with vascular were identified and dissected through membrane dissection method. For unexperienced surgeons, these PGs are easily found superior and inferior. Therefore, these types of PGs are also easily identifiable with integrated dissection.

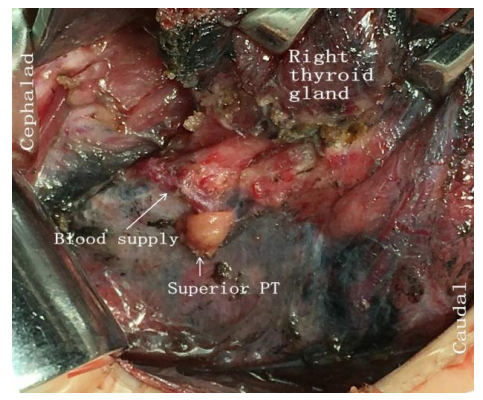

Figure 3. Un-developed superior parathyroid gland (white arrow), un-developed blood supply of parathyroid gland (white arrow). PT: parathyroid gland. The parathyroid gland with vascular was easily identified and dissected. According to the terminal branches' number of PG vascular, It is further identified surrounding structures, especially the main blood vessel for PG. The identification and exposure of PGs usually begin at the inferior vein of the gland by standard inferior approach. For experienced surgeons, these PGs are easily found at the membrane of thyroid gland. Therefore, these types of bifurcation into PGs are also easily identifiable with integrated dissection. PT: parathyroid gland. 


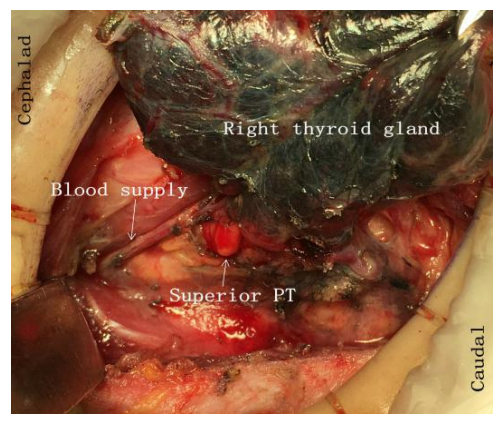

Figure 4. Un-developed right superior parathyroid gland with vasculature (white arrow), Developed right thyroid gland (black). PT: parathyroid gland. The superior PGs' vasculature was characterized by the entry of combing downward branch of posterior arteries and veins from superior vasculature of thyroid glands. The inferior PGs' vasculature was characterized by the entry of combing upward branch of terminal arteries and veins from inferior vasculature of thyroid glands. For experienced surgeons, these PGs could be found superiorly to confirm the combing their vasculature of downward branch of the STA and STV into the PGs and found inferiorly to confirm the combing their vasculature of downward branch of the ITA and ITV into the PGs. The superior pole was ligated and cut close to the thyroid capsule by preserving the posterior branches of superior thyroid artery (STA) for superior PT after the gland developed completely within 10 minutes. The terminal branches of inferior thyroid artery (ITA) were preserved for the inferior PT after ITA cut.

\section{Results}

The average age of the patients was 52.2 years, ranging from 27 to 85 years. The gender ratio was 2:7 (19 males/65 females, Table 1). The PTGs with vasculature were not stained and hence showed the primary yellow color (Figure 3 and Figure 4). In our cases, the staining diffusion time of the non-nodular thyroid lobes was within 5 minutes after injection. In comparison, the staining of the nodular thyroid lobe was not well diffused, and the post-staining diffusion time was more than 5 minutes. All patients had no significant complications and were discharged within 7 days on average. One PTG being seen as adipose tissue or lymph node in the test group was removed, which had been verified by the pathological results, but six PTGs were removed in the control group (Table 2). Six PTGs $(14.29 \%)$ in the control group and one $(2.38 \%)$ in the test group were autotransplanted during thyroidectomy (Table 2 ). Ten patients suffered hypoparathyroidism in the test group at 1 day after surgery and 24 patients of the control group with less than $15 \mathrm{pg} / \mathrm{ml}$ PTH. The hypoparathyroidism numbers were significantly different between the two groups $(23.81 \%$ vs. $51.14 \%, \mathrm{P}<$ $0.05)$, as verified by the $\chi^{2}$ test.

There was no mortality after surgery, and the incidence of transient hypocalcemia was $9.5 \%$ (4 patients) in the test group and $11.9 \%$ (5 patients) in the control group. All patients of hypocalcemia developed transient paresthesia or tetany, lasting up to 3 days (Table 2). Oral calcium and calcitonin capsules were given to improve the hypocalcemia, and subsequently, normal serum PTH levels and hypocalcemia were improved in patients with postoperative 30-day follow-up (Table 2). The occurrence rates of postoperative hypocalcemia were not 
Table 2. Clinical indicators and hypoparathyroidism status of patients.

\begin{tabular}{cccc}
\hline Variable & Nanocarbon particles group & Control group & P value \\
\hline $\begin{array}{c}\text { Parathyroid gland } \\
\text { Incidental remove (n) } \\
\text { Autotransplantation (n) }\end{array}$ & 1 & 6 & \\
$\begin{array}{c}\text { Laboratory results of PTH } \\
\text { (Mean } \pm \text { SD, mg/dl) }\end{array}$ & $1(2.38 \%)$ & $6(14.29 \%)$ & 0.048 \\
Preoperative & & & \\
+1 day & $58.07 \pm 4.17$ & $50.96 \pm 4.29$ & 0.238 \\
+30 day & $28.17 \pm 2.87$ & $19.50 \pm 2.84$ & 0.035 \\
Laboratory results of serum calcium & $47.93 \pm 4.02$ & $38.68 \pm 4.72$ & 0.140 \\
$($ Mean \pm SD, mmol/l) & & & \\
Preoperative & & & \\
+1 day & $2.29 \pm 0.01$ & $2.32 \pm 0.02$ & 0.221 \\
+30 day & $2.14 \pm 0.02$ & $2.19 \pm 0.03$ & 0.197 \\
Transient-hypoparathyroidism (\%) & $2.25 \pm 0.02$ & $2.27 \pm 0.03$ & 0.590 \\
Transient-hypocalcemia (\%) & $10(23.81 \%)$ & $24(51.14 \%)$ & 0.002 \\
\hline & $4(9.5 \%)$ & $5(11.9 \%)$ & 1.000 \\
\hline
\end{tabular}

significantly different in the two groups at 1st day after surgery, as verified by the $\chi^{2}$ test $(9.5 \%$ vs. $11.9 \%, P>0.05)$. In addition, the serum calcium levels were not significantly different between groups $(2.14 \pm 0.02$ vs. $2.19 \pm 0.03, \mathrm{P}>0.05)$, as verified by Student's $\mathrm{t}$ test. But serum PTH levels were significantly higher in the test group than in the control group at 1 day after surgery, according to Student's t test $(28.17 \pm 2.87$ vs. $19.50 \pm 2.84, \mathrm{P}<0.05)$. However, no significant difference in serum PTH levels between the two groups remained at 30 days after surgery (Table 2). Furthermore, the patients were not afflicted with vocal cord paralysis or persistent hypoparathyroidism.

\section{Discussion}

The post-thyroidectomy hypoparathyroidism and hypocalcemia caused a handicap on patients' life with no low incidence, and the rates of transient hypocalcemia and permanent hypocalcemia were reportedly $9.9 \%$ and $4.4 \%$ respectively in a Scandinavian multicenter study [8]. We introduced an easy technique for intraoperative identification of PTGs using ready-made nanocarbon particles. Identifying and localizing PTGs in an accurate, safe and efficient manner are challenging. Methylene blue, a heterocyclic aromatic compound, has been widely used by thyroid surgeons in recent years [9]. After being found to have potential teratogenic effects, methylene blue leading to higher incidence rate of PTG injury in thyroid operation was replaced by the CNPs suspension [10]. Nanocarbon particles were used in intralymph vessels for the negative developed identification of PTGs. Nanocarbon suspension was considered safe for humans, and side effects such as toxicity were not directly related with the volume and con- 
centration used [11]. Nanocarbon suspension is the only lymph tracer approved for clinical application. Nanocarbon particles provide the thyroid surgeon with direct information about the identification and location of PTGs without staining, but thyroid glands and lymph nodes were developed. This technique was adopted in thyroidectomy during the thyroid carcinoma surgery by Hagiwara A et al., who reported the first case series using nanocarbon particle injection for localization and preservation of PTGs [12]. We also tested the adequacy of the identification by using the pathological results of frozen-section or intraoperative PTH determination. As reported, frozen-section diagnosis and intraoperative PTH increase the success rate of PTG preservation in thyroid surgery [13] [14]. The PTGs maintain the original reddish-brown color, and thus can be easily found and distinguished from the black-stained structures such as thyroid tissues and lymph nodes during the surgery. Therefore, the lack-stained tissues as well as surrounding vasculatures should be preserved for their blood supplies (Figure 3 and Figure 4), especially lymph nodes were dissected in thyroid carcinoma operation. The particles of nanocarbon suspension have strong tropism of lymph in diameter of $150 \mathrm{~nm}$. They can pass through the space between the lymph capillary cells in range of $120-150 \mathrm{~nm}$, but cannot enter the blood vessels in range of $20-50 \mathrm{~nm}$ between capillary endothelial cells [4]. As a result, the CNPs will enter the lymphatic vessels after being injected in the peri-tumor thyroid lobe. Then macrophages swallowed nanocarbon particles and entered the lymphatic capillaries, finally accumulating in the lymph nodes and lymphatic vessels. Hence, the thyroid gland and lymph nodes were stained black, but the recurrent laryngeal nerves maintained the original white color, while the vasculatural PTGs remained reddish-brown. Therefore, after being injected into the thyroid tissues, the CNPs will not enter the blood vessels. Instead, they will rapidly enter the lymphatic vessels, then enter the lymphatic capillaries after being internized by macrophages, and finally will accumulate in the lymph nodes, resulting in the black staining of the lymph nodes. Meanwhile, the parathyroids maintain the original reddish-brown and thus are remarkably distinguished from the adjacent black-stained thyroid and lymph nodes. We hypothesize that the difference in the development results is due to the lympho-vascular structure of these tissues. In histology, the lympho-vascular structure of the PTGs is extremely sparse. The recurrent laryngeal nerve was not stained because it was covered by the schwann sheath and had no vascular structure of lymph. According to recent studies and our using experience, CNPs do not cause serious reactions to our body; we recommend that 1- to 2-point peritumoral injections with a dose for each point do not exceed $0.1 \mathrm{ml}$ and a 3- to 5-minute waiting time after the injection of CNPs will be impaired when tumor cells or inflammation blocks lymphatic channels. If the lymph vessels were sparse, the staining result was not diffused well, such as the nodular thyroid lobe. We specifically emphasize that we did the injection just after pulling back to prevent the CNPs injection into blood vessels, and pressed the puncture point for the CNPs not to spill out of the tissues. Some findings in a study cohort show no significant dif- 
ference in the complication rates between total/subtotal thyroidectomy with CNPs injection and that without CNPs injection [15]. The reason was that the identification rate of vascular PTGs was higher in the CNPs staining group, but the successful preservation of PTG function relies on the skills of surgeons. Furthermore, our research showed a significant difference. We firstly used CNPs injection to identify the PTGs with blood supplies from other tissues, combined with the parathyroid vasculature-preserving technique to protect the function of PTGs, and reduced the incidence of postoperative decrease of PTH levels and lower serum calcium. However, the rates of postoperative transient hypoparathyroidism, hypocalcemia, and accidental parathyroid removal were lower in our test group. But a larger number of samples will be accumulated for clarifying this controversy. In conclusion, visual parathyroid identification and its blood supply protection, in respect to parathyroid treatment, have emerged as the "gold standard" of care.

The serum PTH level was less than $15 \mathrm{pg} / \mathrm{ml}$ in 24 patients of the control group (51.14\%) and 10 patients of the test group (23.81\%). Hypocalcemia occurred in 5 patients of the control group (20\%) and 4 patients of the test group $(4 \%)$ at 1 day after surgery. No between-group significant difference was found in hypocalcemia at 1st day, which was different from the results of hypocalcemia at 48 hours after surgery reported by Luo [16]. This was because every patient immediately got calcium supplementation after total thyroidectomy, which affected the calcium serum level at 1 day after surgery. At 30 days after surgery, 2 patients of the control group still exhibited hypocalcemia after the PTG compensation, but no patients of the test group underwent hypocalcemia. The difference of hypoparathyroidism number between the two groups was significant (23.81\% vs. $51.14 \%, \mathrm{P}<0.05)$, as verified by the $\chi^{2}$ test. In addition, serum PTH levels were significantly higher in the test group than in the control group at 1 day after surgery $(28.17 \pm 2.87$ vs. $19.50 \pm 2.84, \mathrm{P}<0.05)$, as indicated by Student's $t$ test. This suggests that PTG damage was significantly reduced in the test group, in which the patients received nanocarbon suspension. The incidental removed numbers and autotransplantation numbers of PTG were more less in the test group, which means the surgeons using the nanocarbon particles made less iatrogenic injuries to the PTG.

There are some limitations in our study. First, the number of female patients was more than three times that of male patients, so we need to improve the number of males in future studies. Second, the necessary steps were not elucidated clearly for protection of PTG with vasculature during thyroid surgery. Hence the incidence rates of hypoparathyroidism were higher in the two groups. Finally, iatrogenic PTGs with the anatomical blood supply were not evaluated. Future multi-institutional studies in larger sample size are needed to clarify whether the lower frequency of post-thyroidectomy hypoparathyroidism relates with the increasing rates of nanocarbon suspension. Despite these limitations, our study has elucidated the safe way of preventing hypoparathyroidism is to identify and dissect vasculatural PTGs by using a ready-made CNPs suspension. 
In conclusion, intraoperative CNPs injection is a safe, useful and easy tool in identification and preservation of vascular PTGs during thyroidectomy, and even inexperienced surgeons can easily identify the recurrent laryngeal nerve, vascular PTG and ITA. CNPs negative localization facilitates the identification and successful preservation of vascular PTGs, even in the reoperative thyroid. There are no systemic complications related to the dye injection. Through identification of PTGs using a negative development technique, the injury of parathyroid blood supply and accidental removal of parathyroids effectively decreased, thus improving the quality of life in patients.

\section{Conclusion}

One major concern for surgeons is to preserve the parathyroid glands with intact structure and undamaged function. In our opinion, the safety for thyroid surgery is based on the protection and visualization of the normal anatomy of parathyroid glands and their blood supplies according to the CNPs injection technique, as verified in our research. This technique can identify the undeveloped parathyroid glands and their blood supplies within 3 - 5 minutes, and recognize the undeveloped recurrent laryngeal nerves and developed lymph nodes. To verify the reliability of CNPs injection, this technique should be applied by more surgeons to study more cases in furture.

\section{Acknowledgements}

The author would like to thank Prof. Bin Song sharing knowledge on PG dissection, available in the dissection skills discussed, and promoting the identification of vasculatural PTGs using CNPs suspension for years now.

\section{Funding}

This study did not receive any fund.

\section{Ethical Approval}

All procedures performed in studies involving human participants were in accordance with the ethical standards of the institutional research committee and with the 1964 Helsinki declaration and its later amendments or comparable ethical standards.

\section{Informed Consent}

Informed consent was obtained from all individual participants included in the study.

\section{Authors' Contributions}

Study conception and design: Chuanchang Yin, Bin Song.

Acquisition of data: Xiaoyan Wang, Chuanchang Yin.

Analysis and interpretation of data: Xiaoyan Wang. 
Drafting of manuscript: Chuanchang Yin.

Critical revision of manuscript: Xiaoyan Wang.

\section{Conflicts of Interest}

Author Chuanchang Yin declares that he has no conflict of interest. Author Xiaoyan Wang declares that she has no conflict of interest. Author Bin Song declares that she has no conflict of interest.

\section{References}

[1] Li, Y. (2015) A Meta-Analysis of Carbon Nanoparticles for Identifying Lymph Nodes and Protecting Parathyroid Glands during Surgery. Otolaryngology-Head and Neck Surgery, 152, 1007-1016. https://doi.org/10.1177/0194599815580765

[2] Zarebczan, B. and Chen, H. (2011) Influence of Surgical Volume on Operative Failures for Hyperparathyroidism. Advances in Surgery, 45, 237-248. https://doi.org/10.1016/j.yasu.2011.03.003

[3] Shinden, Y., et al. (2017) Intraoperative Identification of the Parathyroid Gland with a Fluorescence Detection System. World Journal of Surgery, 41, 1506-1512. https://doi.org/10.1007/s00268-017-3903-0

[4] Li, J., Li, X. and Wang, Z. (2013) Negative Developing of Parathyroid Using Carbon Nanoparticles during Thyroid Surgery. Gland Surgery, 2, 100-101.

[5] Abboud, B., Sargi, Z., Akkam, M. and Sleilaty, F. (2002) Risk Factors for Post Thyroidectomy Hypocalcaemia. Journal of the American College of Surgeons, 195, 456-461. https://doi.org/10.1016/S1072-7515(02)01310-8

[6] Stack, B.C., Bimston, D.N., Bodenner, D.L., et al. (2015) American Association of Clinical Endocrinologists and American College of Endocrinology Disease State Clinical Review: Postoperative Hypoparathyroidism-Definitions and Management. Endocrine Practice, 21, 674-685. https://doi.org/10.4158/EP14462.DSC

[7] Shoback, D.M., Bilezikian, J.P., Costa, A.G., et al. (2016) Presentation of Hypoparathyroidism: Etiologies and Clinical Features. The Journal of Clinical Endocrinology and Metabolism, 101, 2300-2312. https://doi.org/10.1210/jc.2015-3909

[8] Bergenfelz, A., Jansson, S., Kristoffersson, A., Martensson, H., Reihner, E., Wallin, G. and Lausen, I. (2008) Complications to Thyroid Surgery: Results as Reported in a Database from a Multicenter Audit Comprising 3,660 Patients. German Langenbeck's Archives of Surgery, 393, 667-673.

https://doi.org/10.1007/s00423-008-0366-7

[9] Harari, A., Sippel, R.S., Goldstein, R., Aziz, S., Shen, W., Gosnell, J. and Clark, O.H. (2012) Successful Localization of Recurrent Thyroid Cancer in Reoperative Neck Surgery Using Ultrasound-Guided Methylene Blue Dye Injection. Journal of the American College of Surgeons, 215, 555-561. https://doi.org/10.1016/j.jamcollsurg.2012.06.006

[10] Liu, F., Zhu, Y., Qian, Y., Zhang, J., Zhang, Y. and Zhang, Y. (2017) Recognition of Sentinel Lymph Nodes in Patients with Papillary Thyroid Cancer by Nano-Carbon and Methylene Blue. Pakistan Journal of Medical Sciences, 33, 1485-1489. https://doi.org/10.12669/pjms.336.13940

[11] Sippel, R.S., Elaraj, D.M., Poder, L., et al. (2009) Localization of Recurrent Thyroid Cancer Using Intraoperative Ultrasound-Guided Dye Injection. World Journal of Surgery, 33, 434-439. https://doi.org/10.1007/s00268-008-9797-0 
[12] Hagiwara, A., Takahashi, T., Sawai, K., et al. (1992) Lymph Nodal Vital Staining with Newer Carbon Particle Suspensions Compared with India Ink: Nanocarbon Particles and Clinical Observations. Lymphology, 25, 84-89.

[13] Herrera, A. (1999) Parathyroid Autotransplantation during Thyroidectomy: Is Frozen Section Necessary? Journal of Oral and Maxillofacial Surgery, 57, 1474. https://doi.org/10.1016/S0278-2391(99)90741-3

[14] Edafe, O., Antakia, R., Laskar, N., Uttley, L. and Balasubramanian, S.P. (2014) Systematic Review and Meta-Analysis of Predictors of Post-Thyroidectomy Hypocalcaemia. British Journal of Surgery, 101, 307-320. https://doi.org/10.1002/bjs.9384

[15] Liu, X., Chang, S., Jiang, X., Huang, P. and Yuan, Z. (2016) Identifying Parathyroid Glands with Carbon Nanoparticle Suspension Does Not Help Protect Parathyroid Function in Thyroid Surgery. Surgical Innovation, 23, 381-389.

https://doi.org/10.1177/1553350615624787

[16] Luo, D. (2014) Application of Nano-Carbon in Lymph Node Dissection for Thyroid Cancer and Protection of Parathyroid Glands. Medical Science Monitor, 20, 1925-1930. https://doi.org/10.12659/MSM.890721 